\title{
Stress Wave Propagation and Energy Absorption Properties of Heterogeneous Lattice Materials under Impact Load
}

\author{
Cun Zhao, Meng Zhang ${ }^{D}$, Guoxi Li, and Dong Wang \\ College of Intelligent Science, National University of Defense Technology, Changsha, China \\ Correspondence should be addressed to Meng Zhang; zhangmengchn@126.com
}

Received 22 October 2021; Accepted 10 December 2021; Published 23 December 2021

Academic Editor: Hao Yi

Copyright (c) 2021 Cun Zhao et al. This is an open access article distributed under the Creative Commons Attribution License, which permits unrestricted use, distribution, and reproduction in any medium, provided the original work is properly cited.

\begin{abstract}
A heterogeneous lattice material composed of different cells is proposed to improve the energy absorption capacity. The heterogeneous structure is formed by setting layers of body-centered XY rods (BCC $x y$ ) cells as the reinforcement in the bodycentered cubic (GBCC) uniform lattice material. The heterogeneous lattice samples are designed and processed by additive manufacturing technology. The stress wave propagation and energy absorption properties of heterogeneous lattice materials under impact load are analyzed by finite element simulation (FES) and Hopkinson pressure bar (SHPB) experiments. The results show that, compared with the GBCC uniform lattice material, the spreading velocity of the stress of the (GBCC)3(BCCxy)2 heterogeneous lattice material is reduced by $18.1 \%$, the impact time is prolonged $27.9 \%$, the stress peak of the transmitted bar is reduced by $34.8 \%$, and the strain energy peak is reduced by $29.7 \%$. It indicates that the heterogeneous lattice materials are able to reduce the spreading velocity of stress and improve the energy absorption capacity. In addition, the number of layers of reinforcement is an important factor affecting the stress wave propagation and energy absorption properties.
\end{abstract}

\section{Introduction}

In recent years, with the increasing maturity of additive manufacturing technology, the development of lattice materials has been greatly promoted. Lattice material is a kind of artificial periodic material with good lightweight, porosity, and designability $[1,2]$. By adjusting the topology configuration, cell sizes and cell arrangement rules, the mechanical properties of lattice materials can be improved to adapt to different engineering applications [3-5]. With the development of the study, it is found that lattice materials include many other important functional potentials, such as cushioning, vibration attenuation, energy absorption, heat dissipation, noise reduction, and electromagnetic shielding, which are expected to be widely used in aerospace, transportation, machinery, and many others fields [6-9]. The energy absorption properties of lattice materials are the focus of our attention.

To explore the energy absorption properties of lattice materials, a series of research studies have been carried out. In 2012, Cui et al. [10] proposed a theoretical model based on the displacement mechanism of lattice structure to predict the dynamic response of square lattice Sandwich plate under impact loading and obtained the stressstrain curve of materials. In 2012, Narisetti et al. [11] proposed a general harmonic balance method for studying plane wave propagation in strongly nonlinear periodic media and found the law of amplitude-dependent dispersion in single cell, double cell, and hexagonal closely arranged two-dimensional lattice structures. In 2013, Shen et al. [12] gave the analytical expression of the dynamic compression response of gradient porous metal structure according to one-dimensional shock wave theory and suggested that the weakest part of the gradient structure should be placed at the impact end to obtain the maximum energy absorption effect. In 2014, Zhao [13] and Karamooz Ravari et al. [14] studied a finite element model to predict the impact change of compression bar diameter on concrete collapse stress by beam and solid finite elements. In 2015, Asadpoure and Valdevit [15] and Qiu et al. [16] studied the relationship between the size, morphology, and internal porosity of lattice materials and 
found that the displacement was uniform before the peak stress under compressive load. In 2016, Mukherjee et al. [17] and Zheng et al. [18] studied theoretically and numerically the one-dimensional impact response and energy absorption of gradient lattice materials with different density distributions. In 2017, Novak et al. [19], Kim et al. [20], and Choy et al. [21] designed and studied the displacement and stress laws of cubic lattice structure and honeycomb lattice structures with different diameters and densities and found that the strain instability of lattice structures always starts from the thinnest layer to the densest layer. In 2018, Hani et al. [22] and Bhattacharyya and Maurice [23] studied the evolution law of interfacial stress in different multilayer dissimilar materials. In 2018, Babaei and Levitas [24] and Zhang et al. [25] studied the influence of temperature field, thermal effect, and other factors on the stress wave behavior of lattice materials, especially the law of directional wave propagation and energy flow based on Bloch's theorem and finite element method. In 2019, Zhu et al. [26] and Zhang et al. [27] studied the propagation law of elastic waves in layered lattice materials. The results show that the lattice structures at different levels have stable bandgap behavior and the bandgap width increases significantly in the reentry hierarchy. In 2020, Isaenkova et al. [28] and Guo et al. [29] studied the displacement process of the lattice structure, and the obtained stress-strain curve parameters show that this structure has anisotropic behavior. In 2020, Karličić et al. [30] and Zhang et al. [31] studied the stress wave propagation of lattice structures. Dynamic simulation shows that the added mass, prestress, and material stiffness have a significant impact on the stress wave and eigenvalues of the lattice structures.

Several research studies show that the energy absorption properties of lattice materials are mainly dominated by cell configuration, cell size, and cell arrangement. For example, pure GBCC lattice materials have good bearing performance but poor energy absorption capacity, and pure BCCxy lattice materials have better energy absorption potential [32-36]. The load conditions are often complex in real applications. The bearing capacity, cushioning performance, and energy absorption properties of the materials should be considered to obtain lightweight lattice materials with good comprehensive performance. Furthermore, in the future, with the help of additive manufacturing technology, there are more abundant changes in cell configuration and cell arrangement of lattice materials. Therefore, it is of great significance to combine GBCC cells and BCCxy cells to form heterogeneous lattice materials and study the stress propagation and energy absorption properties of heterogeneous lattice materials.

This paper is organized as follows. In Section 2, the heterogeneous lattice materials are introduced. In Section 3, the stress wave propagation and energy absorption properties in heterogeneous lattice materials are analyzed by simulation. In Section 4, the lattice samples and the Hopkinson pressure bar system are introduced in detail. In Section 5, the experimental results are analyzed and discussed. Finally, the conclusions are given in Section 6.

\section{Materials and Methods}

2.1. Heterogeneous Lattice Materials. The composite structure is shown in Figure 1(a). The composite structure is mainly composed of matrix phase and reinforcement. The matrix phase bears the external force to protect the reinforcement. The reinforcement improves the functional properties, such as cushioning, energy absorption, and heat dissipation [35].

With reference to the composite structure, the heterogeneous lattice materials are proposed as shown in Figure 1. The cells with stable geometric structure and greater equivalent stiffness are arranged in the matrix layer to improve the bearing properties, and the cells with functional potential are arranged in the reinforcement layer to improve the energy absorption properties. Studies show that the body-centered cubic (GBCC) cell has good structural stability and flexural properties [36]. Compared with the GBCC cells, the straight bars in the $z$-direction of the body-centered $X Y$ bar (BCC $x y)$ cells are removed. The BCC $x y$ cell is more prone to displacement to resist external forces and absorb energy, which indicates a better energy absorption potential. The heterogeneous lattice material composed of GBCC cells and BCC $x y$ cells is recorded as (GBCC) $i(\mathrm{BCC} x y) j$, abbreviated as $\mathrm{GiBj}$. I and $j$ correspond to the number of layers of the matrix layer and reinforcement layer. The (GBCC) $4(\mathrm{BCC} x y) 1$ heterogeneous lattice model is shown in Figure 1(c), abbreviated as G4B1. The (GBCC)3(BCC $x y) 2$ heterogeneous lattice model is shown in Figure $1(\mathrm{~d})$, abbreviated as G3B2.

2.2. The Spreading Velocity of Stress. The concept of stress wave was first put forward by S-D (Poisson et al. in 1930). The stress generates and propagates outward in the form of waves in materials [37]. Just like the amplitude of the wave that will gradually decrease, the stress will be weakened during propagation.

The spreading velocity of stress is as follows [38]:

$$
\left\{\begin{array}{l}
v_{E}=v_{m}+(1+\varepsilon) v_{0}, \\
v_{0}=\left(\frac{E}{\rho_{0}}\right)^{1 / 2},
\end{array}\right.
$$

where $v_{m}$ is the particle velocity, $v_{0}$ is the spreading velocity of stress, $\sigma$ is the engineering stress, $\varepsilon$ is the engineering strain, $E$ is the elastic modulus, and $\rho_{0}$ is the density.

According to the laws of mass conservation and momentum conservation, a quasilinear wave equation with displacement $u(t, x)$ is established as follows [39]:

$$
\frac{\partial^{2} u}{\partial t^{2}}-v_{0}^{2} \frac{\partial^{2} u}{\partial x^{2}}=0
$$

It can be seen from (1) and (2) that the spreading velocity of stress is related to the density, elastic modulus, displacement rate, and other properties of the material.

It is assumed that the spreading velocity of stress in different lattice layers is different and can be superimposed. 


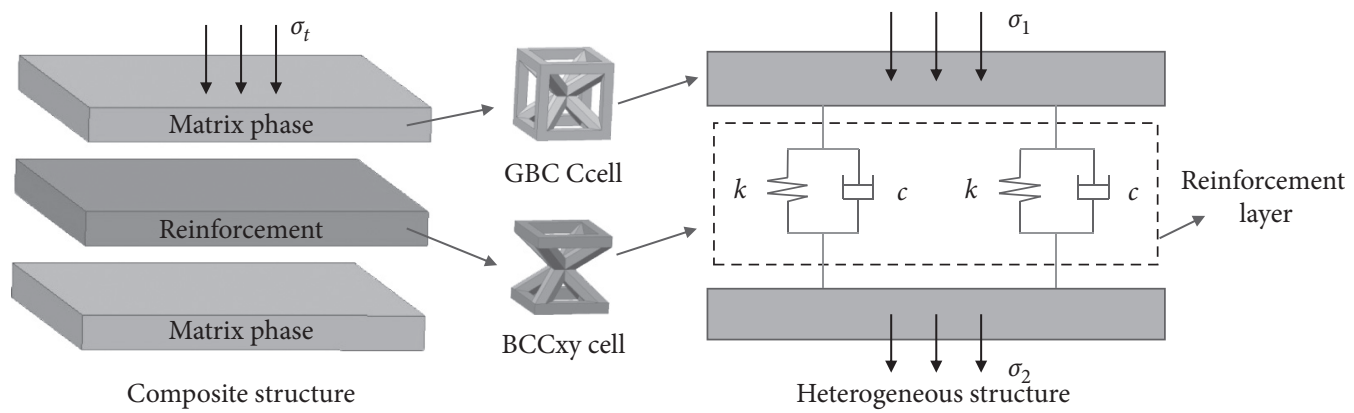

(a)

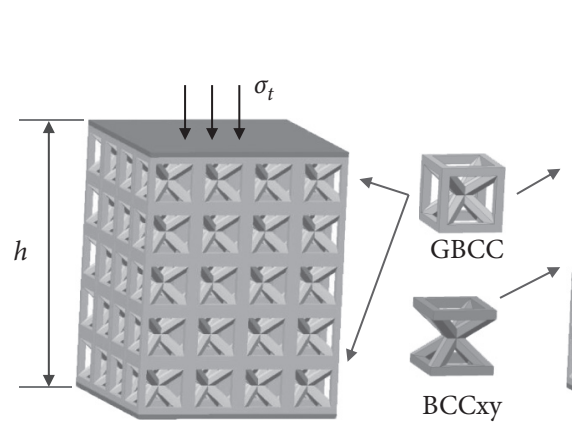

(b)

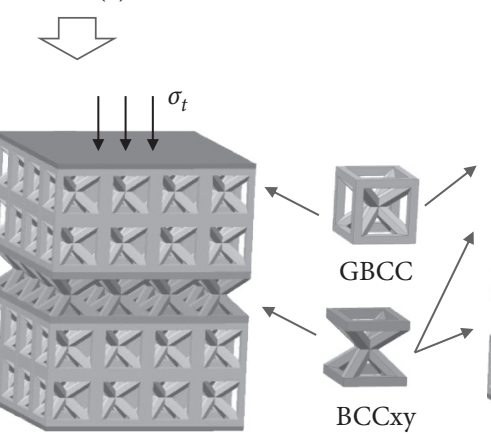

(c)

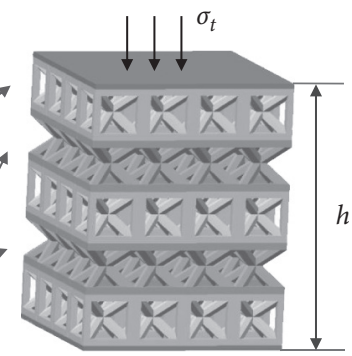

(d)

Figure 1: Material model: (a) composite structure, (b) GBCC uniform lattice material, (c) G4B1 heterogeneous lattice material, and (d) G3B2 heterogeneous lattice material.

The approximate formula of the average velocity of stress wave propagation $v_{i j}$ in heterogeneous lattice materials is as follows:

$$
v_{i j}=\frac{v_{1} v_{2}(i+j)}{i v_{1}+j v_{2}}+\Delta,
$$

where $v_{1}$ and $v_{2}$ are the spreading velocity of stress in the GBCC and BCC $x y$ uniform lattice materials and $\Delta$ is a small quantity, which can be ignored in the approximate analysis.

According to the above analysis, the spreading velocity of stress in $\mathrm{G} i \mathrm{~B} j$ heterogeneous lattice material is greater than the GBCC uniform lattice material. The spreading velocity of stress in heterogeneous lattice materials is determined by the number of layers of matrix phase and reinforcement.

\subsection{The Strain Energy of Heterogeneous Lattice Materials.} According to the elastic-plastic theory of materials, the lattice cell can be equivalent to a spring-damping element [39]. The work done by the external force is mainly offset by the bending displacement of bars in the cell to play the role of bearing, cushioning, and energy absorption [40].

The cell models are shown in Figure 2. The structural parameters of the cell are composed of side size $a$ and member section size $b$. GBCC cell produces strain energy through the compression displacement of the straight bar and bending displacement of the inclined bar, and BCC xy cell produces strain energy mainly through the bending displacement of the inclined bar.

Under the same load, the elastic parameters are as follows:

$$
\left\{\begin{array}{l}
\varepsilon_{1}<\varepsilon_{2}, \\
E_{1}>E_{2}, \\
u_{1}<u_{2}, \\
k_{1}>k_{2} .
\end{array}\right.
$$

The work done by the external force is as follows [41]:

$$
\left\{\begin{array}{l}
W_{F}=\int_{0}^{\Delta u} F \cdot \mathrm{d} u, \\
Q_{e}=\int_{0}^{\varepsilon_{m}} \sigma \cdot \mathrm{d} \varepsilon, \\
W_{F}=Q_{e},
\end{array}\right.
$$

where $W_{F}$ is the work done by the external force, $Q_{e}$ is the strain energy of a single cell, and $F$ is the external force.

The equivalent strain energy of cells is as follows:

$$
\left\{\begin{array}{l}
Q_{e 1}=\frac{F^{2} a}{4 E b_{1}^{2}} \cdot\left(C_{1}+C_{2} \sqrt{\frac{1}{3}+\frac{512 a^{4}}{75 b_{1}^{4}}}\right), \\
Q_{e 2}=\frac{F^{2} a}{4 E b_{2}^{2}} \cdot \sqrt{\frac{1}{3}+\frac{512 a^{4}}{75 b_{2}^{4}}}
\end{array}\right.
$$

where $C_{1}$ and $C_{2}$ are dimensionless coefficients, $a$ is the side size of cells, and $b_{1}$ and $b_{2}$ are the sizes of member sections.

The parameter relationship is as follows: 


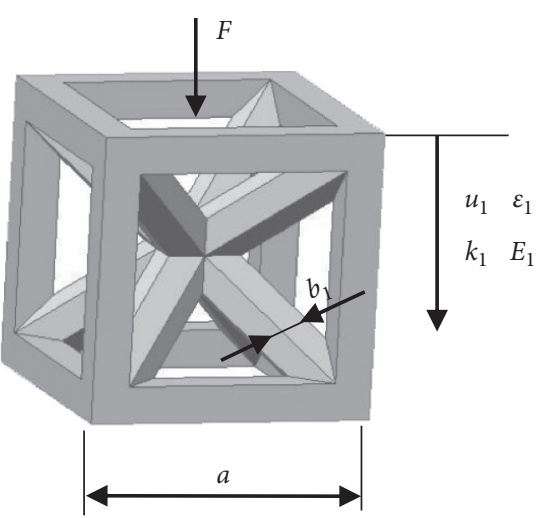

(a)

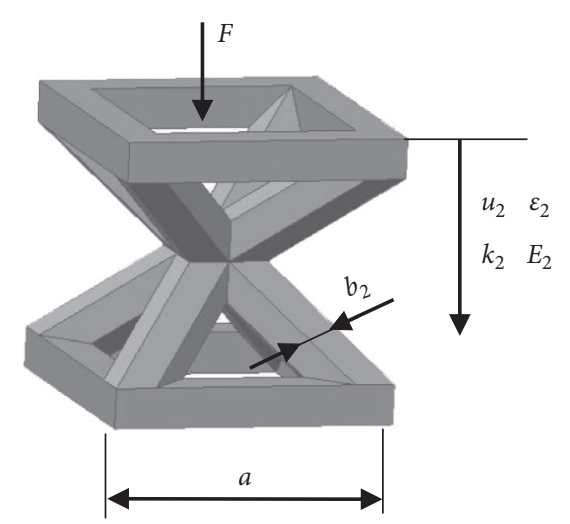

(b)

Figure 2: Cell models: (a) GBCC cell and (b) BCCxy cell.

$$
\left\{\begin{array}{l}
b_{1}<b_{2}, \\
k_{1}>k_{2}, \\
Q_{e 1}<Q_{e 2}, \\
0<C_{2}<C_{1}<1 .
\end{array}\right.
$$

It shows that the lattice materials composed of GBCC cells have better bearing capacity and the lattice materials composed of BCCxy cells have better energy absorption capacity. The equivalent strain energy of the lattice material is approximately the superposition of the strain energy of each layer of cells. The strain energy of lattice materials is as follows:

$$
\left\{\begin{array}{l}
Q_{\mathrm{GBCC}}=\sum_{1}^{n} \sum_{1}^{m} \sum_{1}^{s} \mathrm{Q}_{e 1}, \\
\mathrm{Q}_{\mathrm{GiBj}}=\sum_{1}^{i} \sum_{1}^{m} \sum_{1}^{s} \mathrm{Q}_{e 1}+\sum_{1}^{j} \sum_{1}^{m} \sum_{1}^{s} \mathrm{Q}_{e 2} \\
\mathrm{Q}_{\mathrm{GBCC}}<\mathrm{Q}_{\mathrm{GiBj}}
\end{array}\right.
$$

where $Q_{\mathrm{GBCC}}$ is the the strain energy of the GBCC uniform lattice material, $Q_{G i B j}$ is the strain energy of the $G_{i B j}$ heterogeneous lattice material, $Q_{e 1}$ and $Q_{e 2}$ are the strain energy of a single cell, $s, m$, and $n$ correspond to the number of cells in each direction, and $i$ and $j$ are the number of the matrix layers and reinforcement layers, $n=i+j$.

According to the above analysis, pure GBCC cells have good bearing performance, and pure BCCxy lattice materials are expected to have greater energy absorption potential. However, the equivalent stiffness of BCCxy cells is small. The combination of BCCxy cells and other cells with good bearing performance (such as GBCC cells) will be a new application way. The strain energy of the $\mathrm{GiBj}$ heterogeneous lattice material is greater than the GBCC uniform lattice material. It indicates that the energy absorption capacity of the $\mathrm{GiBj}$ heterogeneous lattice materials is better than the GBCC uniform lattice material. In other words, the energy absorption properties of the uniform lattice materials can be improved by setting the reinforcement layers composed of BCCxy cells.

\section{The Finite Element Simulation of Lattice Materials}

3.1. Simulation Model. The 3D models of the uniform lattice material and heterogeneous lattice materials are established with GBCC cells and BCCxy cells. The given design domain is $w \times l \times h, w=40 \mathrm{~mm}, l=40 \mathrm{~mm}$, and $h=50 \mathrm{~mm}$. The side size of cells is $a, a=10 \mathrm{~mm}$. The cell number is $s \times m \times n$. The weight loss ratio is $\lambda, \lambda=70 \%$, the relative density $r$ is determined, $r=0.3$. The size of member section $b$ is determined by $a$ and $r$. The parameters of the four lattice material models are shown in Table 1. The 3D model of the GBCC uniform lattice material is shown in Figure 1(b). The 3D model of the BCC $x y$ uniform lattice is similar to the GBCC uniform lattice. The 3D models of the G4B1 and G3B2 heterogeneous lattice materials are shown in Figures 1(c) and 1(d). The G4B1 heterogeneous lattice material is with a layer of reinforcement, and the G3B2 heterogeneous lattice material is with two layers of reinforcement. A $1 \mathrm{~mm}$ diaphragm is added to the lattice material to maintain the flatness.

The dynamic simulation model of lattice materials under impact load is established, as shown in Figure 3(a). The simulation model is mainly composed of the incident bar, lattice materials, and the transmitted bar. The incident bar hits the left (top) of the lattice material along the $x$-axis. The right (bottom) of the lattice material is supported by the transmitted bar. The velocity of the incident bar is $v_{0}$, $v_{0}=5 \mathrm{~m} / \mathrm{s}$. The displacement, stress, strain, and strain energy are collected to analyze the stress wave propagation and energy absorption properties of lattice materials under impact load by simulation.

3.2. Displacement and Impact Time. The simulation results are shown in Figure 3. The displacements of the left end of lattice materials and the transmitted bar are shown in Figures 3(b) and 3(c). When $t_{0}=0.00005 \mathrm{~s}$, the incident bar strikes the lattice materials, the lattice materials begin to produce compression displacement, and the displacement curve rises gradually to reach the peak. 
TABLE 1: Parameters of the lattice material models.

\begin{tabular}{|c|c|c|c|c|c|c|c|}
\hline \multirow[t]{2}{*}{ Number } & \multirow[t]{2}{*}{ Lattice type } & \multirow[t]{2}{*}{ Number of cells $s \times m \times n$} & \multirow[t]{2}{*}{ Weight loss ratio $\lambda(\%)$} & \multirow[t]{2}{*}{ Relative density $r$} & \multirow[t]{2}{*}{ Side size $a(\mathrm{~mm})$} & \multicolumn{2}{|c|}{$\begin{array}{c}\text { Member section } \\
\text { size }\end{array}$} \\
\hline & & & & & & $b_{1}(\mathrm{~mm})$ & $b_{2}(\mathrm{~mm})$ \\
\hline 1 & GBCC & $4 \times 4 \times 5$ & 70 & 0.3 & 10 & 1.06 & - \\
\hline 2 & G4B1 & $4 \times 4 \times 5$ & 70 & 0.3 & 10 & 1.06 & 1.16 \\
\hline 3 & G3B2 & $4 \times 4 \times 5$ & 70 & 0.3 & 10 & 1.06 & 1.16 \\
\hline 4 & BCCxy & $4 \times 4 \times 5$ & 70 & 0.3 & 10 & - & 1.16 \\
\hline
\end{tabular}

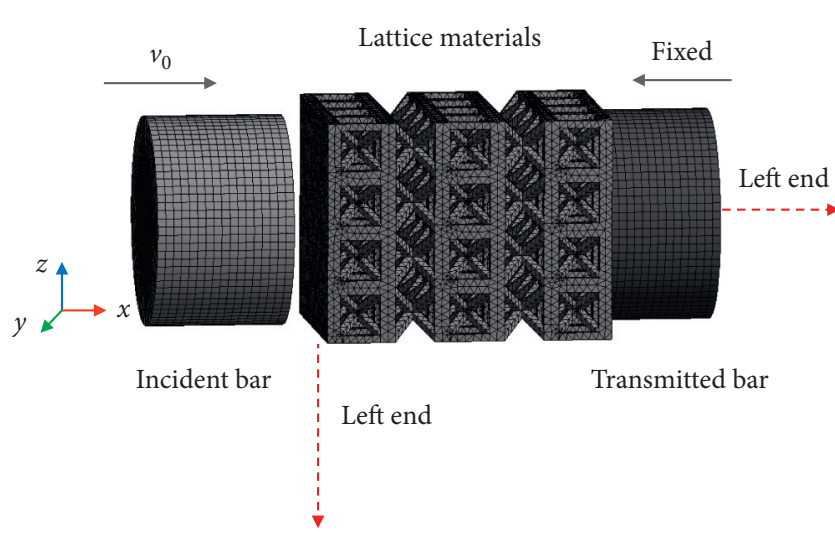

(a)

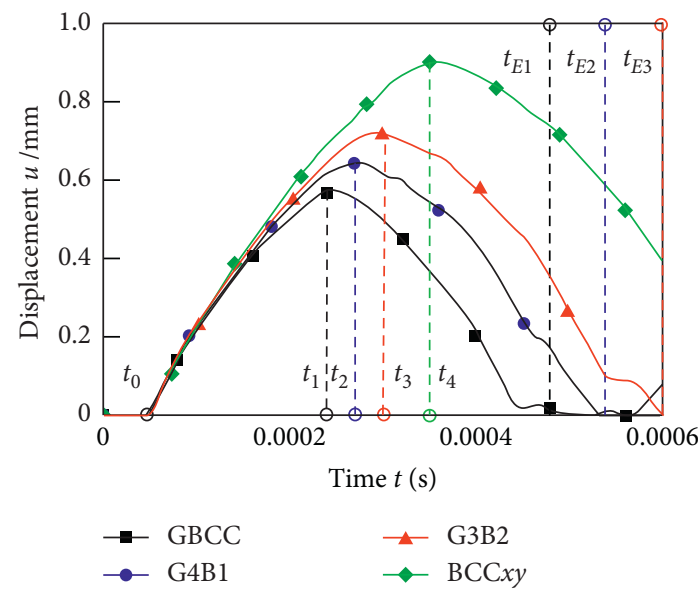

(c)

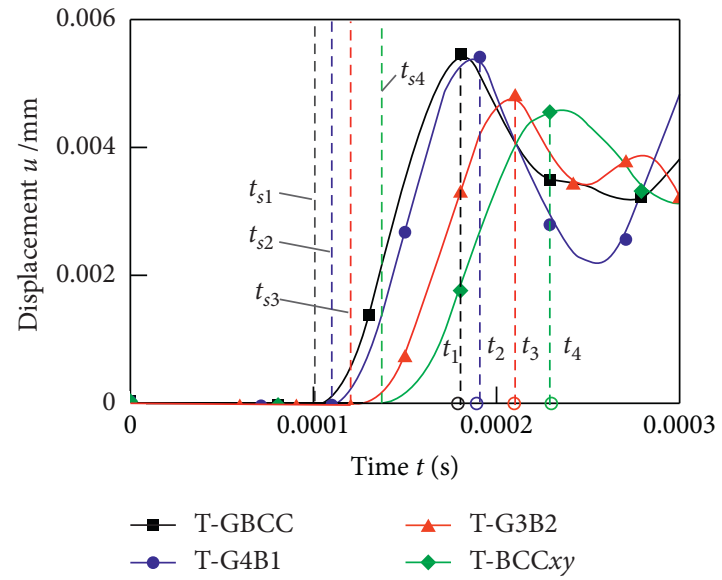

(b)

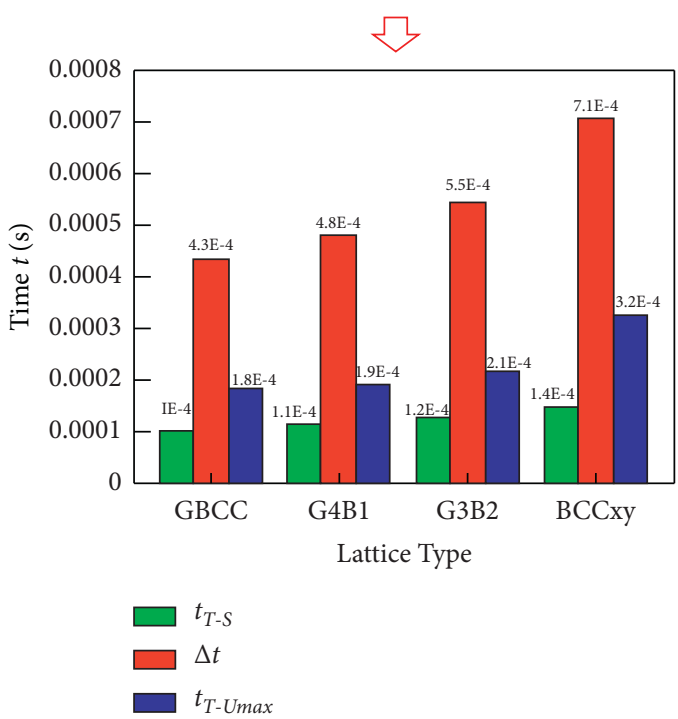

(d)

Figure 3: Simulation results: (a) simulation model, (b) displacement of samples, (c) displacement of the transmitted bar, and (d) impact time.

As shown in Figure 3(a), the maximum displacement of the three lattice materials is as follows: $u_{\mathrm{GBCC}}=0.57 \mathrm{~mm}$, $u_{\mathrm{G} 4 \mathrm{~B} 1}=0.65 \mathrm{~mm}, u_{\mathrm{G} 3 \mathrm{~B} 2}=0.72 \mathrm{~mm}$, and $u_{\mathrm{BCC} x y}=0.90 \mathrm{~mm}$. Compared with the GBCC uniform lattice material, the maximum displacement of the G4B1 and G3B2 heterogeneous lattice materials is increased by $14.1 \%$ and $26.3 \%$. Compared with the GBCC uniform lattice material, the maximum displacement of the BCC xy uniform lattice material is increased by $57.8 \%$.
The displacement curve of the transmitted bar is shown in Figure 3(b). The maximum displacement of the transmitted bar is as follows: $u_{T \text {-GBCC }}=0.0057 \mathrm{~mm}, u_{T \text {-G } 4 \mathrm{~B} 1}=0.0054 \mathrm{~mm}$, $u_{T \text {-G3B2 }}=0.0048 \mathrm{~mm}$, and $u_{T \text {-BCC } x y}=0.0045 \mathrm{~mm}$. Compared with the GBCC uniform lattice material, the maximum displacement of the transmitted bar of the G4B1 and G3B2 heterogeneous lattice materials is reduced by $5.3 \%$ and $15.7 \%$. The simulation results show that, compared with the GBCC uniform lattice material, the G4B1 and G3B2 heterogeneous 
lattice materials can effectively reduce the displacement of the transmitted bar under impact load.

The impact time is shown in Figure 3(c). The starting time of impact displacement of the transmitted bar is $t_{0}$, $t_{0}=0.00005 \mathrm{~s}$. The time to reach the maximum displacement of the three lattice materials is as follows: $t_{1}=0.00025 \mathrm{~s}$, $t_{2}=0.00027 \mathrm{~s}, t_{3}=0.00030 \mathrm{~s}$, and $t_{4}=0.00035 \mathrm{~s}$. The end time of impact displacement of lattice materials is as follows: $t_{\mathrm{E} 1}=0.00048 \mathrm{~s}, t_{\mathrm{E} 2}=0.00053 \mathrm{~s}$, and $t_{\mathrm{E} 3}=0.00060 \mathrm{~s}$. As shown in Figure 3(b), the starting time of impact displacement of the transmitted bar is as follows: $t_{\mathrm{S} 1}=0.0001 \mathrm{~s}$, $t_{\mathrm{S} 2}=0.00011 \mathrm{~s}, t_{\mathrm{S} 3}=0.00012 \mathrm{~s}$, and $t_{\mathrm{S} 4}=0.00014 \mathrm{~s}$. The time to reach the maximum displacement is as follows: $t_{1}=0.00018 \mathrm{~s}, t_{2}=0.00019 \mathrm{~s}, t_{3}=0.00021 \mathrm{~s}$, and $t_{4}=0.00032 \mathrm{~s}$. As shown in Figure $3(\mathrm{~d})$, the impact time of three lattice materials is as follows: $\Delta t_{\mathrm{GBCC}}=0.00043 \mathrm{~s}, \Delta t_{\mathrm{G} 4 \mathrm{~B} 1}=$ $0.00048 \mathrm{~s}, \Delta t_{\mathrm{BCC} x y}=0.00071 \mathrm{~s}$, and $\Delta t_{\mathrm{G} 3 \mathrm{~B} 2}=0.00055 \mathrm{~s}$.

Compared with the GBCC uniform lattice material, the starting time of impact displacement of the transmitted bar of the G4B1 and G3B2 heterogeneous lattice materials is prolonged by $10 \%$ and $20 \%$, and the impact time is prolonged by $11.6 \%$ and $27.9 \%$. Compared with the G4B1 heterogeneous lattice materials, the starting time of impact displacement of the transmitted bar of the G3B2 lattice materials is prolonged by $9.1 \%$, and the impact time is prolonged by $14.5 \%$. The simulation results show that, compared with the GBCC uniform lattice material, the G4B1 and G3B2 heterogeneous lattice materials can effectively prolong the impact time to buffer and protect the transmission rod.

In addition, the results show that, among the four lattice material models, the BCC $x y$ uniform lattice material has the best effect on prolonging the impact time and the best cushioning potential. However, under the same impact load, the BCCxy uniform lattice material has greater displacement, which reflects its poor bearing performance and is prone to local deformation and failure. The application of pure BCCxy lattice materials is greatly limited.

In the following, the stress propagation and energy absorption properties of GBCC, G4B1, and G3B2 lattice materials will be studied in detail.

3.3. The Law of Stress Wave Propagation. The stress nephogram of lattice materials by finite element simulation is shown in Figure 4(a). It can be seen from Figure 4(a) that the maximum stress concentration areas of different lattice materials are different. During the impact process, the maximum stress, time, and position data of lattice materials are extracted for further analysis. The stress-time curves and position-time curves of the stress peak are shown in Figures 4(b) and 4(c).

It can be seen from Figure 4(b) that the stress of lattice materials gradually reaches the peak and then decreases. The stress peaks of different lattice materials are different. The stress peaks of three lattice materials are as follows: $\sigma_{\mathrm{GBCC}}=34.01 \mathrm{MPa}, \quad \sigma_{\mathrm{G} 4 \mathrm{~B} 1}=38.93 \mathrm{MPa}, \quad$ and $\sigma_{\mathrm{G} 3 \mathrm{~B} 2}=$ 35.35 $\mathrm{MPa}$. The time to reach the stress peaks of the three lattice materials that are different is as follows: $t_{1}=0.00018 \mathrm{~s}$, $t_{2}=0.00024 \mathrm{~s}$, and $t_{3}=0.00029 \mathrm{~s}$. Compared with the GBCC uniform lattice material, the time to reach the stress peak of the G4B1 and G3B2 heterogeneous lattice materials is significantly prolonged by $33.3 \%$ and $61.1 \%$. Compared with the G4B1 heterogeneous lattice materials, the time to reach the stress peak of the G3B2 heterogeneous lattice materials is prolonged by $20.8 \%$.

It can be seen from Figure 4(c) that, under the impact load, the stress peak in the lattice material is transmitted back and forth from the left to right of the material and the amplitude decreases gradually. The stress wave propagation in lattice materials shows obvious periodic fluctuation and damping vibration trend. The function of the stress wave $f(\sigma$, $x, t)$ is defined as follows:

$$
f(\sigma, x, t) \Longrightarrow\left\{\begin{array}{l}
\sigma(t)=\sigma_{\max } y(t), \\
x(t)=\mathrm{Ae}^{-\beta t} \sin (\mathrm{wt}+\phi),
\end{array}\right.
$$

where $\sigma(t)$ is a stress-time function, $\sigma_{\max }$ is the maximum stress, $y(t)$ is the gradual change function, $x(t)$ is the position-time function of the stress peak, $A$ is the displacement amplitude, and $\beta$ is the damping factor.

As shown in Figure 4(c), the periods of the stress wave are as follows: $T_{1}=0.00018 \mathrm{~s}, T_{2}=0.00020 \mathrm{~s}, T_{3}=0.00022 \mathrm{~s}$, and $T_{1}<T_{2}<T_{3}$. Compared with the BCC $x y$ uniform lattice, the period of the stress wave in the G3B2 and G4B1 heterogeneous lattice materials is increased by $11.1 \%$ and $22.2 \%$. The simulation results show that, compared with the GBCC uniform lattice material, the G4B1 and G3B2 heterogeneous lattice materials can effectively prolong the stress wave period.

The stress of the transmitted bar is shown in Figure 5. The stress peaks of the transmitted bar are as follows: $\sigma_{T-\mathrm{GBCC}}=63.61 \mathrm{MPa}, \sigma_{T-\mathrm{G} 4 \mathrm{~B} 1}=61.08 \mathrm{MPa}, \sigma_{T-\mathrm{G} 3 \mathrm{~B} 2}=55.94 \mathrm{MPa}$, and $\sigma_{T-\mathrm{G} 3 \mathrm{~B} 2}<\sigma_{T \text {-G4B1 }}<\sigma_{T-G B C C}$. Compared with the GBCC uniform lattice material, the stress peaks of the transmitted bar of the G4B1 and G3B2 heterogeneous lattice materials are reduced by $3.9 \%$ and $12.1 \%$. Compared with the G4B1 heterogeneous lattice material, the stress peaks of the transmitted bar of the G3B2 heterogeneous lattice materials are reduced by $8.4 \%$. The simulation results show that the G4B1 and G3B2 heterogeneous lattice materials can effectively reduce the stress peak of the transmitted bar. And the optimization effect of the heterogeneous lattice material with two layers of reinforcement is better than that with one layer.

As shown in Figure 5, the rise time of stress of the transmitted bars is as follows: $t_{\mathrm{S} 1}=0.00009 \mathrm{~s}, t_{\mathrm{S} 2}=0.00010 \mathrm{~s}$, $t_{\mathrm{S} 3}=0.00011 \mathrm{~s}$, and $t_{\mathrm{S} 1}<t_{\mathrm{S} 2}<t_{\mathrm{S} 3}$. The time to reach the stress peaks of the transmitted bars is as follows: $t_{1}=0.00018 \mathrm{~s}, t_{2}=0.00024 \mathrm{~s}, t_{3}=0.00029 \mathrm{~s}$, and $t_{1}<t_{2}<t_{3}$. The starting time of impact is $t_{0}, t_{0}=0.00005 \mathrm{~s}$. The time of stress transfer to the transmitted bars is as follows: $\Delta t_{1}=0.00004 \mathrm{~s}, \quad \Delta t_{2}=0.00005 \mathrm{~s}, \Delta t_{3}=0.00006 \mathrm{~s}$, and $\Delta t_{1}<\Delta t_{2}<\Delta t_{3}$. Compared with the GBCC uniform lattice material, the time of stress transfer to the transmitted bars of the G4B1 and G3B2 heterogeneous lattice materials is prolonged by $25 \%$ and $50 \%$. 

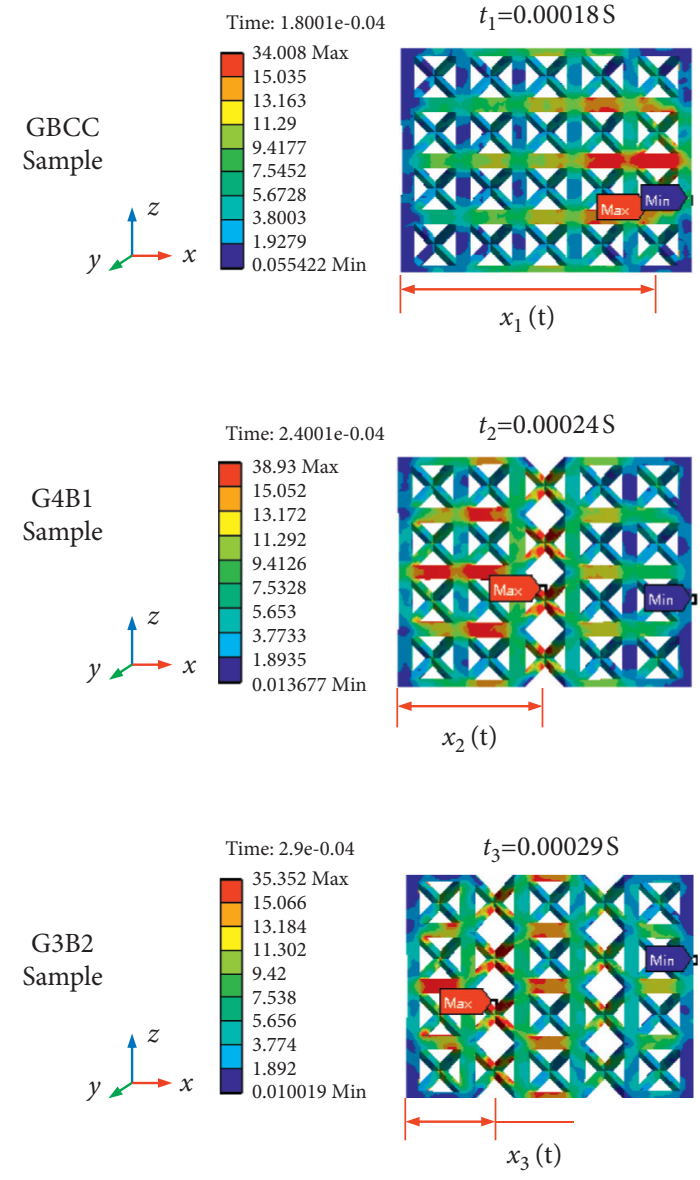

(a)

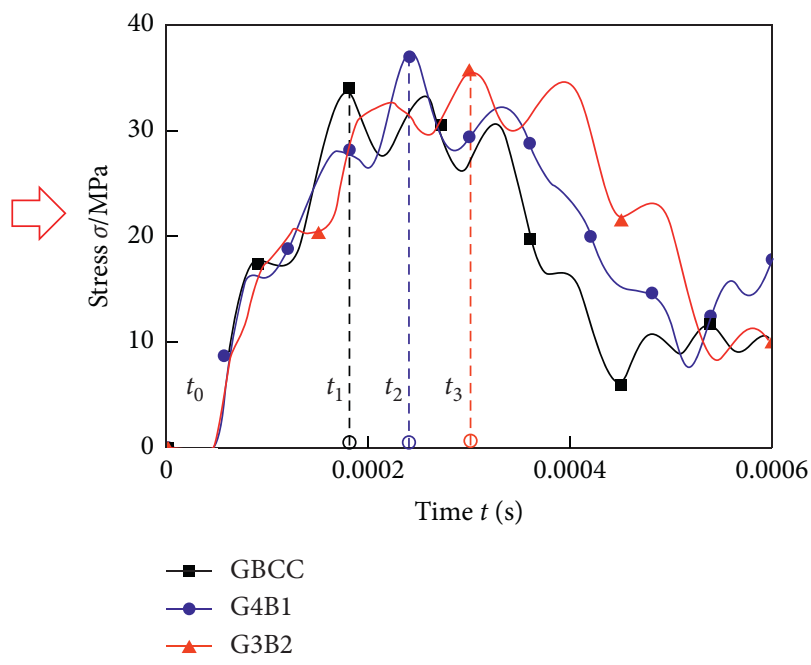

(b)

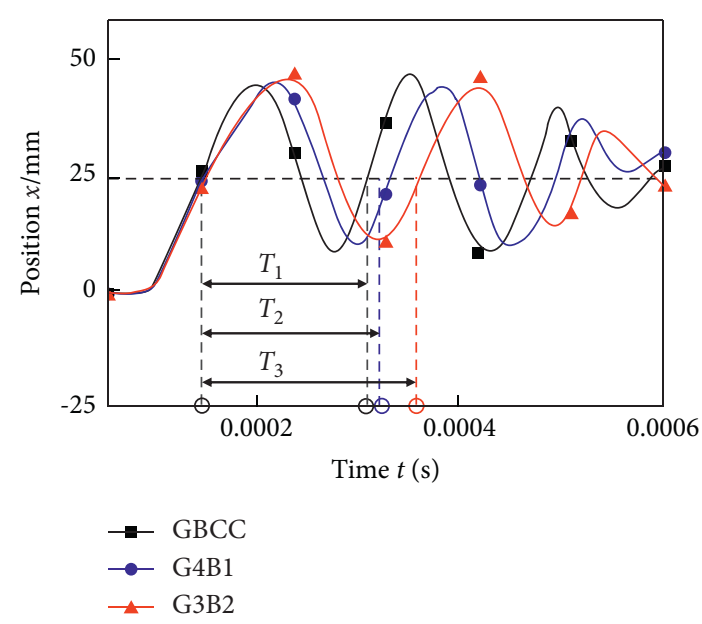

(c)

Figure 4: Stress of lattice materials: (a) stress nephogram, (b) stress-time curves, and (c) position-time curves of stress peak.

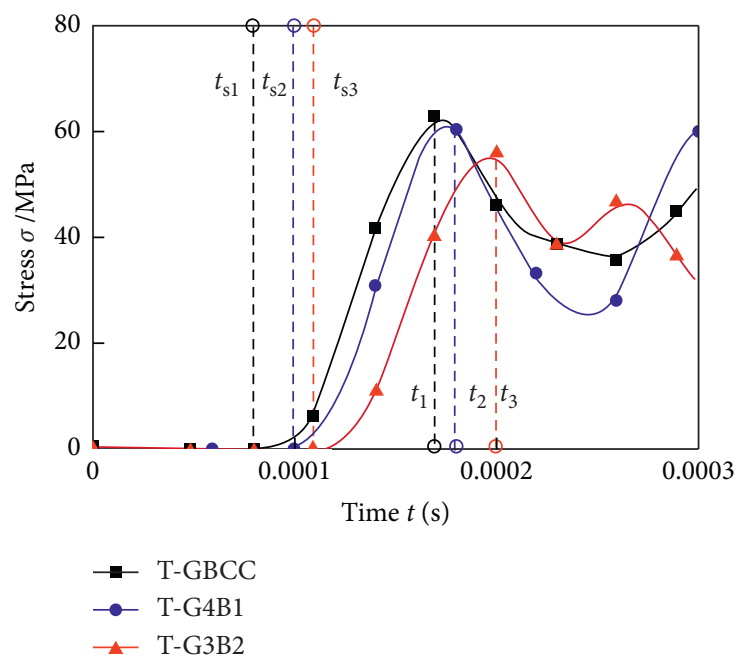

FIGURE 5: The stress of the transmitted bar. 
According to the time of stress transfer to the transmitted bars and the thickness of the lattice material, the spreading velocity of stress is calculated as shown in Figure 6 . The spreading velocity of stress in the three lattice materials is as follows: $v_{\mathrm{GBCC}}=1300 \mathrm{~m} / \mathrm{s}, v_{\mathrm{G} 4 \mathrm{~B} 1}=1040 \mathrm{~m} / \mathrm{s}$, $v_{\mathrm{G} 3 \mathrm{~B} 2}=866 \mathrm{~m} / \mathrm{s}$, and $v_{\mathrm{G} 3 \mathrm{~B} 2}<v_{\mathrm{G} 4 \mathrm{~B} 1}<v_{\mathrm{GBCC}}$. According to the position-time function $x(t)$ and the period of the stress wave, the spreading velocity of the stress peak is calculated as shown in Figure 6. The average velocities of the stress peaks in the three lattice materials are as follows: $v_{\mathrm{GBCC}}=577 \mathrm{~m} / \mathrm{s}$, $v_{\mathrm{G} 4 \mathrm{~B} 1}=520 \mathrm{~m} / \mathrm{s}, v_{\mathrm{G} 3 \mathrm{~B} 2}=472 \mathrm{~m} / \mathrm{s}$, and $v_{\mathrm{G} 3 \mathrm{~B} 2}<v_{\mathrm{G} 4 \mathrm{~B} 1}<v_{\mathrm{GBCC}}$.

Compared with the GBCC uniform lattice material, the spreading velocity of stress wave of the G4B1 and G3B2 heterogeneous lattice materials is reduced by $20 \%$ and $33.3 \%$, and the spreading velocity of stress peak is reduced by $9.8 \%$ and $18.1 \%$. Compared with the G4B1 heterogeneous lattice material, the spreading velocity of stress of the G3B2 heterogeneous lattice material is reduced by $16.7 \%$, and the spreading velocity of stress peak is reduced by $9.2 \%$.

The simulation results show that, compared with the GBCC uniform lattice material, the G4B1 and G3B2 heterogeneous lattice materials can effectively reduce the spreading velocity of stress. Furthermore, compared with the G4B1 heterogeneous lattice materials, the spreading velocity of stress of the G3B2 heterogeneous lattice materials is slower. It indicates that the number of layers of reinforcement is an important factor affecting the propagation of stress.

3.4. Energy Absorption Properties. In the whole impact process, part of the impact energy is absorbed by the lattice materials, and the remaining energy is transferred to the transmitted bar. According to the energy conversation law, the greater the energy absorbed by the lattice materials, the smaller the energy absorbed by the transmission rod. The strain energy is used as the evaluation index of energy absorption properties of lattice materials. The strain energy can be calculated from the stress-strain integral. The strain energy curves of lattice materials and the transmission rod are shown in Figure 7.

As shown in Figure 7(a), the strain energy peaks of the three lattice materials are as follows: $Q_{1}=76.1 \mathrm{~mJ}$, $Q_{2}=92.3 \mathrm{~mJ}, Q_{3}=97.9 \mathrm{~mJ}$, and $Q_{1}<Q_{2}<Q_{3}$. Compared with the GBCC uniform lattice material, the strain energy peak of the G4B1 and G3B2 heterogeneous lattice materials is increased by $21.3 \%$ and $28.6 \%$. Compared with the G4B1 heterogeneous lattice material, the strain energy peak of the G3B2 heterogeneous lattice materials is increased by $6.1 \%$. As shown in Figure $7(\mathrm{~b})$, the strain energy peaks of the transmitted bar are as follows: $Q_{1}=18.7 \mathrm{~mJ}, Q_{2}=16.5 \mathrm{~mJ}$, and $Q_{3}=13.1 \mathrm{~mJ}, Q_{3}<Q_{2}<Q_{1}$. Compared with the GBCC uniform lattice material, the strain energy peak of the transmitted bar of the G4B1 and G3B2 heterogeneous lattice materials is reduced by $11.7 \%$ and $29.9 \%$. Compared with the G4B1 heterogeneous lattice material, the strain energy peak of the transmitted bar of the G3B2 heterogeneous lattice materials is reduced by $20.6 \%$.

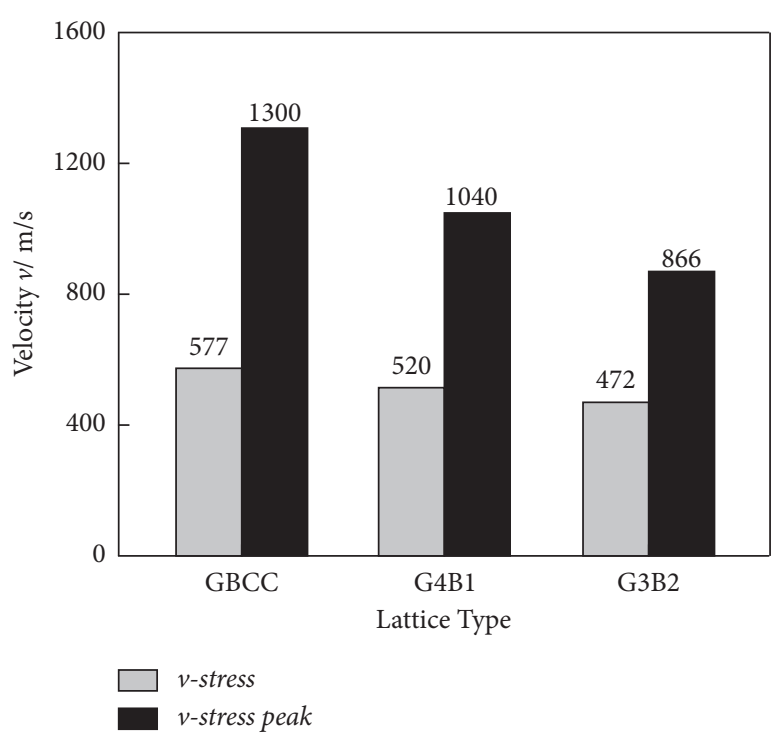

FIGURE 6: The average velocity of stress wave in lattice materials.

The simulation results show that the heterogeneous lattice materials can effectively improve the energy absorption capacity. Furthermore, the energy absorption capacity of the heterogeneous lattice materials with two layers of reinforcement is better than that with a layer of reinforcement. It indicates that the number of layers of reinforcement is an important factor affecting the energy absorption properties of heterogeneous lattice materials.

\section{Experiment}

4.1. Samples. According to the $3 \mathrm{D}$ models of the three lattice materials, the samples are processed by stereolithography appearance (SLA) additive manufacturing technology. The processing equipment is the Ultra H500 $3 \mathrm{D}$ printer, and the forming range is $500 \mathrm{~mm} \times 500 \mathrm{~mm} \times 800 \mathrm{~mm}$; the printing thickness is $0.05 \mathrm{~mm} \sim 0.4 \mathrm{~mm}$, and the forming accuracy is $0.05 \mathrm{~mm}$. The material is Nylon PA12, density $\rho=0.95 \mathrm{~g} / \mathrm{cm}^{-3}$, elastic modulus $E=1.8 \mathrm{GPa}$, and Poisson's ratio $v=0.33$.

The GBCC uniform lattice sample and G4B1 and G3B2 heterogeneous lattice samples are shown in Figures 8(b)$8(\mathrm{~d})$. The geometric structure of the samples is consistent with the $3 \mathrm{D}$ models of lattice materials.

4.2. Impact Testing System. The Hopkinson pressure bar (SHPB) system is used to carry out the impact test of lattice samples, as shown in Figure 8(a). As shown in Figure 8(a), the bullet, incident bar, transmitted bar, and absorption bar are made of aluminum alloy, with an elastic modulus of $71 \mathrm{GPa}$ and a diameter of $\Phi 38 \mathrm{~mm}$ with lengths of $600 \mathrm{~mm}$, $1600 \mathrm{~mm}, 1200 \mathrm{~mm}$, and $800 \mathrm{~mm}$, respectively. Strain gauges 1 and 2 adopt the kd3000 strain sensor with the measurement range of $0 \sim 200 \mathrm{kN}$ and charge sensitivity of $1.93 \mathrm{pC} / \mathrm{N}$. KD6009 strain amplifier and MDO3014 oscilloscope are used to enhance the signals. The sampling frequency is $5 \mathrm{MHz}$ and the number of sampling points is 10000 . The 


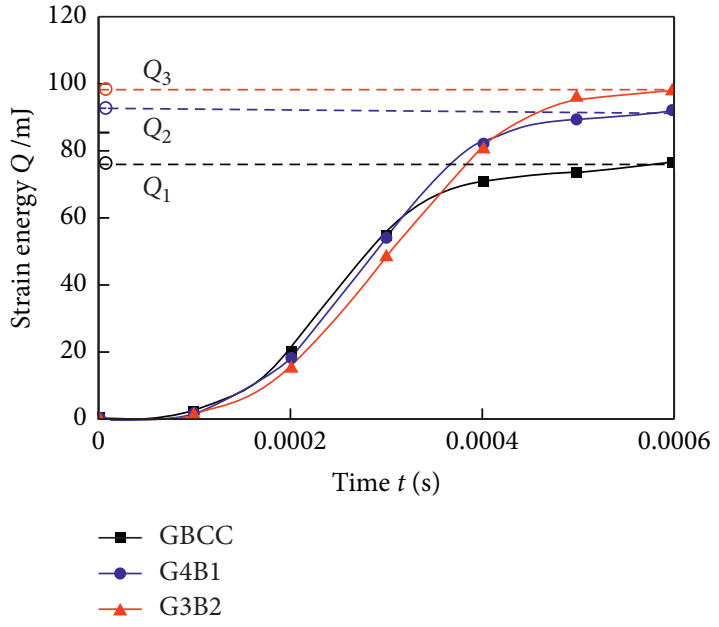

(a)

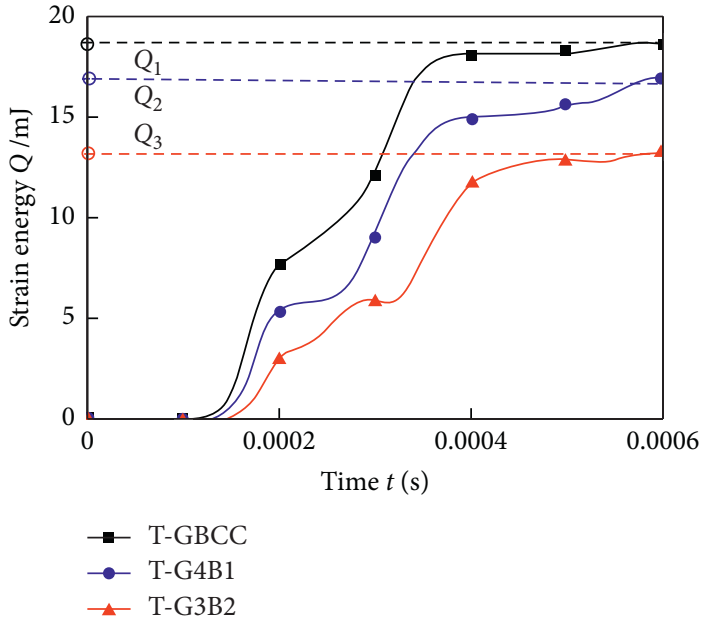

(b)

FIGURE 7: Strain energy curve: (a) lattice model and (b) transmitted bar.

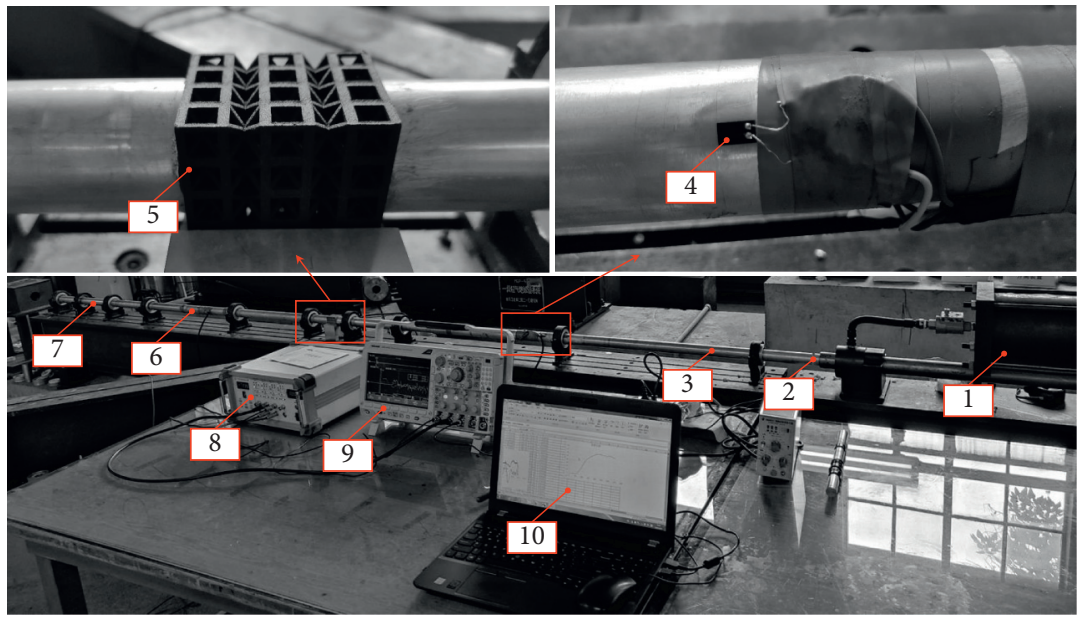

(a)

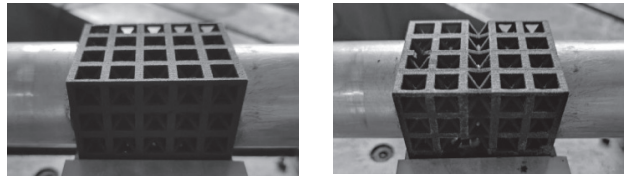

(b)

(c)

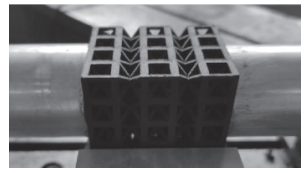

(d)

Figure 8: The experiment system and samples: (a) SHPB system: (1) air pump, (2) bullet, (3) incident bar, (4) strain gauge, (5) test piece, (6) transmitted bar, (7) absorber bar, (8) strain amplifier, (9) oscilloscope, and (10) computer; (b) GBCC sample; (c) G4B1 sample; and (d) G3B2 sample.

stress is recorded by strain gauges 1 and 2 pasted on the incident bar and transmitted bar, and the generated voltage signal is transmitted and stored in the computer. The initial experimental parameters are shown in Table 2.

\section{Results and Discussion}

5.1. Voltage Signal. In the experiment, the pressure of the air pump is set to the same value to ensure the same impact load on the three lattice samples, $P_{0}=0.5 \mathrm{MPa}$. The stress on the incident bar and transmitted bar is collected by strain gauges 1 and 2 , which are recorded as voltage signals $U_{I}$ and $U_{T}$. The incident signals of the three lattice samples are coincident, $U_{I}=0.8 \mathrm{~V}$. The rise time of the voltage signal of the incident bar is $t_{0}, t_{0}=0.00025 \mathrm{~s}$.

The experimental results are shown in Figure 9. It can be seen from Figure 9(a) that the voltage signals collected by the strain gauge 2 on the transmitted bar of three lattice samples are significantly different. The red wireframe marks the first period of the voltage signal. In the first period, the voltage 
TABLE 2: Experimental parameters.

\begin{tabular}{lcccc}
\hline Pressure $P(\mathrm{MPa})$ & Voltage $U_{I}(\mathrm{~V})$ & Sampling frequency $f(\mathrm{MHz})$ & Sensitivity coefficient $K_{1}$ & Amplification factor $K_{1}$ \\
\hline 0.5 & 0.8 & 5 & 1.93 & 100
\end{tabular}

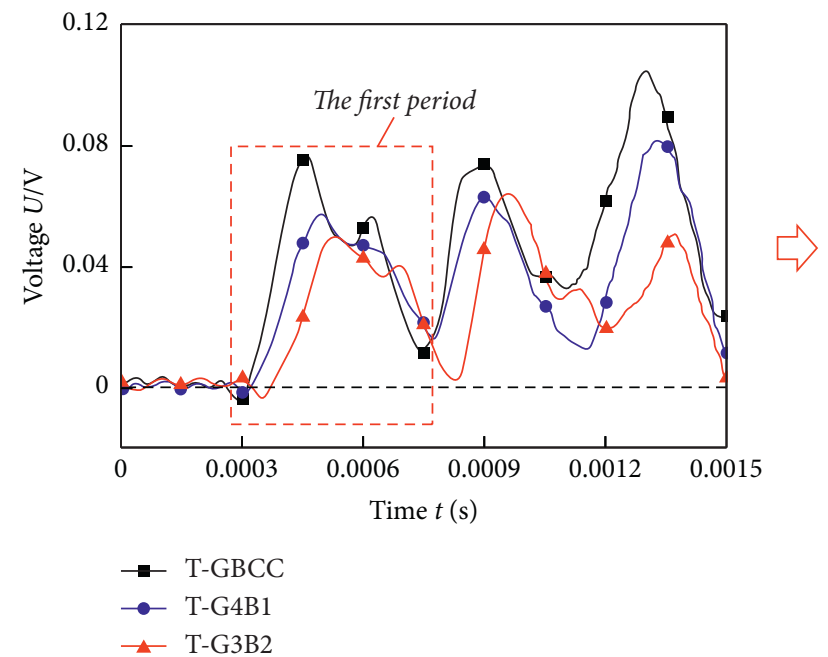

(a)

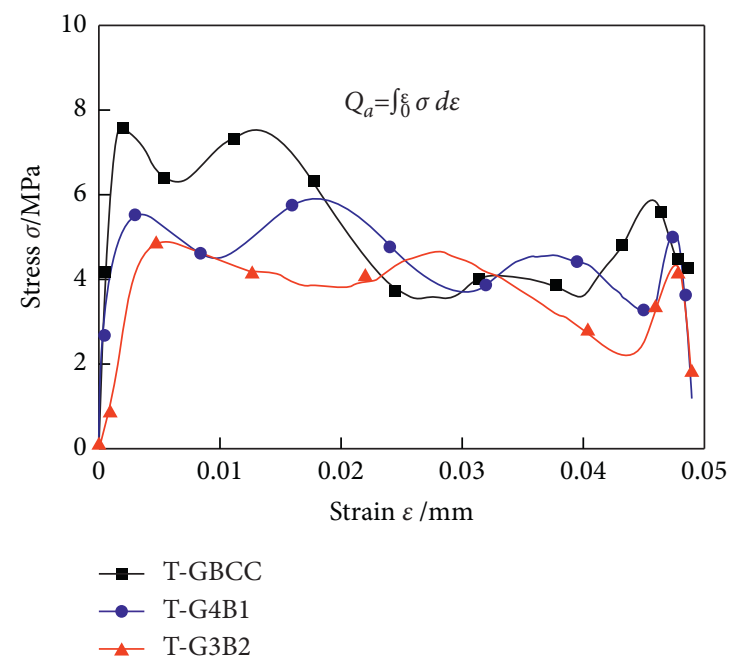

(c)

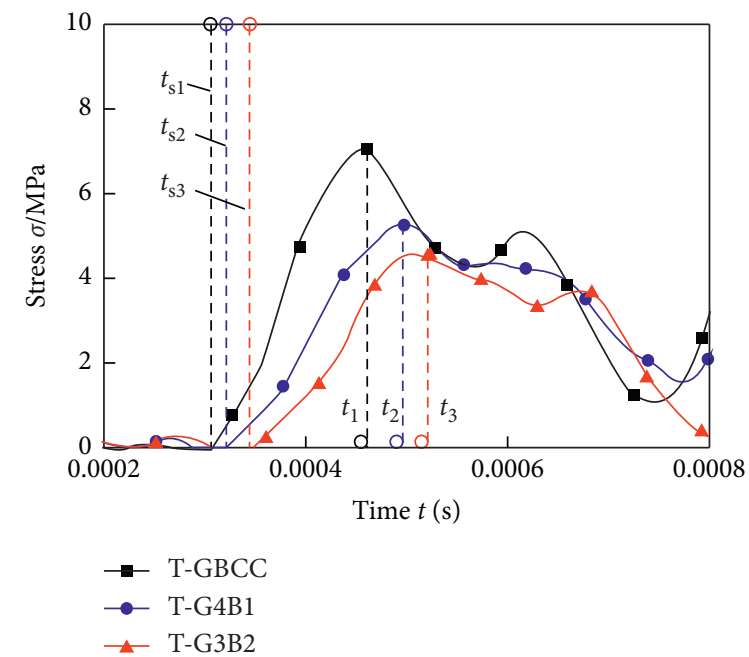

(b)

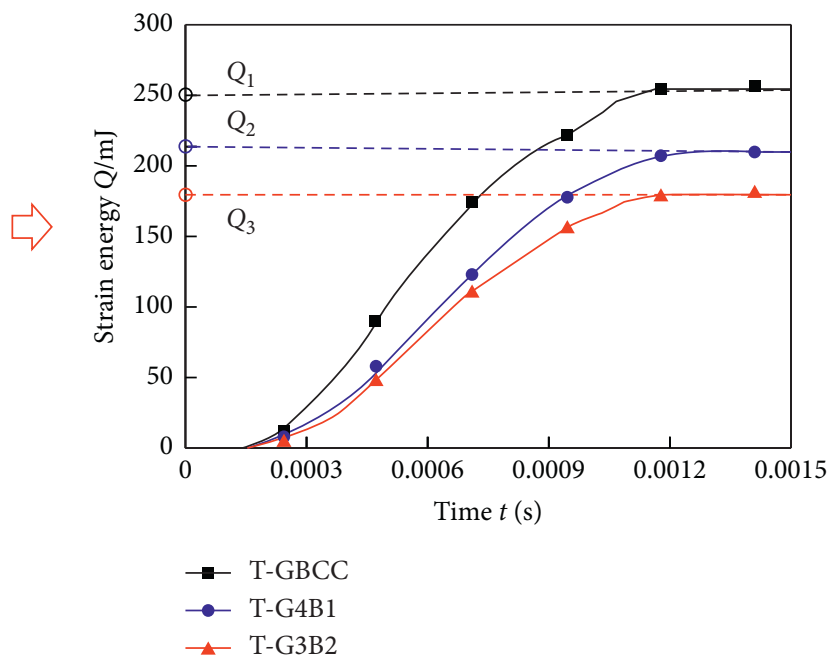

(d)

Figure 9: Experimental results: (a) voltage signal of the transmitted signal, (b) strain curve, (c) stress-strain curve, and (d) strain energy curve.

peaks of the transmitted bar are as follows: $U_{T-\mathrm{GBCC}}=0.0773 \mathrm{~V}$, $U_{T \text {-G4B1 }}=0.0595 \mathrm{~V}$, and $U_{T \text {-G3B2 }}=0.0508 \mathrm{~V}$. It is found that the voltage peak of the transmitted bar of the GBCC uniform lattice sample is the largest and that of the G3B2 heterogeneous lattice sample is the smallest. The different stress signals indicate that the three lattice samples have different impact responses.

5.2. Stress and Strain. The strain signal is measured by the resistance strain gauge method. The stress and strain are calculated according to the voltage signals collected by the strain gauge. Assume that, during the impact process, the incident wave $\varepsilon_{I}$, the reflected wave $\varepsilon_{R}$, and transmitted wave $\varepsilon_{T}$ propagate independently in the compression bar and the force of the sample is balanced. The linear relationship of the three elastic waves is as follows:

$$
\varepsilon_{R}=\varepsilon_{I}-\varepsilon_{T}
$$

According to the linear superposition principle of elastic waves, assuming that the displacement process of the sample is an ideal uniform state, the average strain formula of the sample is as follows [42]: 


$$
\varepsilon_{a}(t)=-\frac{2 c_{0}}{l_{0}} \int_{0}^{t} \varepsilon_{R} \mathrm{~d} t,
$$

where $c_{0}$ is the velocity of the elastic wave in the compression bar and $l_{0}$ is the original length of the sample.

According to the principle of Wheatstone bridge circuit, the strain formula of the bar is as follows [42]:

$$
\varepsilon_{b}(t)=\frac{2 \Delta U(t)}{K_{1} K_{2} U_{0}},
$$

where $K_{1}$ is the sensitivity factor of the strain gauge, $K_{2}$ is the amplification factor of the strain amplifier, $\Delta U(t)$ is the voltage increase measured by the strain gauge, and $U_{0}$ is the supply voltage of DC power.

The stress-strain formula is as follows [42]:

$$
\sigma(t)=\frac{A E}{A_{0}} \varepsilon_{t}
$$

where $A$ is the cross-sectional area of the compression bar, $A_{0}$ is the cross-sectional area of the sample, $E$ is the elastic modulus of the transmitted bar, and $\varepsilon_{t}$ is the strain of the transmitted bar. The stress and strain of the transmitted bar are calculated according to the above formulas.

The stress curve of the transmitted bar is shown in Figure 9(b). It can be seen that the trend of the stress curve is consistent with the voltage curves. The time to reach the stress peak of the transmitted bar is as follows: $t_{1}=0.00046 \mathrm{~s}, t_{3}=0.00049 \mathrm{~s}, t_{4}=0.00052 \mathrm{~s}$, and $t_{1}<t_{2}<t_{3}$. The stress peaks of the transmitted bar are as follows: $\sigma_{\mathrm{T}}$ $\mathrm{GBCC}=7.03 \mathrm{MPa}, \sigma_{T-\mathrm{G} 4 \mathrm{~B} 1}=5.29 \mathrm{MPa}, \sigma_{T-\mathrm{G} 3 \mathrm{~B} 2}=4.58 \mathrm{MPa}$, and $\sigma_{T-\mathrm{G} 3 \mathrm{~B} 2}<\sigma_{T-\mathrm{G} 4 \mathrm{~B} 1}<\sigma_{T-\mathrm{GBCC}}$. Compared with the GBCC uniform lattice material, the stress peaks of the transmitted bar of the G4B1 and G3B2 heterogeneous lattice materials are reduced by $24.7 \%$ and $34.8 \%$. Compared with the G4B1 heterogeneous lattice material, the stress peak of the transmitted bar of the G3B2 heterogeneous lattice sample is reduced by $13.4 \%$. The comparison between Figures 5 and 9(b) shows that the experimental results are consistent with the simulation results, which proves the optimization trend of stress. The results show that, compared with the GBCC uniform lattice material, the G4B1 and G3B2 heterogeneous lattice materials can effectively reduce the stress peak of the transmitted bar.

The starting time of the stress curve of the transmitted bar is as follows: $t_{\mathrm{S} 1}=0.00031 \mathrm{~s}, t_{\mathrm{S} 2}=0.00032 \mathrm{~s}$, $t_{\mathrm{S} 3}=0.00033 \mathrm{~s}$, and $t_{\mathrm{S} 1}<t_{\mathrm{S} 2}<t_{\mathrm{S} 3}$. The time of stress transfer to the transmitted bar is as follows: $\Delta t_{1}=0.00006 \mathrm{~s}$, $\Delta t_{2}=0.00007 \mathrm{~s}, \Delta t_{3}=0.00008 \mathrm{~s}$, and $\Delta t_{1}<\Delta t_{2}<\Delta t_{3}$. Compared with the GBCC uniform lattice material, the time of stress transfer to the transmitted bar of the G4B1 and G3B2 heterogeneous lattice materials is prolonged by $16.6 \%$ and $33.3 \%$. Compared with the G4B1 heterogeneous lattice material, the time of stress transfer to the transmitted bar of the G3B2 heterogeneous lattice material is prolonged by $14.2 \%$. The results show that, compared with GBCC uniform lattice material, the G4B1 and G3B2 heterogeneous lattice materials can effectively prolong the time of stress transfer to the transmitted bar.
According to the time of stress transfer to the transmitted bars and the thickness of the lattice sample, the spreading velocity of stress is calculated as shown in Figure 10. The average velocities of the stress waves in the three lattice samples are as follows: $v_{\mathrm{GBCC}}=866 \mathrm{~m} / \mathrm{s}$, $v_{\mathrm{G} 4 \mathrm{~B} 1}=742 \mathrm{~m} / \mathrm{s}, v_{\mathrm{G} 3 \mathrm{~B} 2}=650 \mathrm{~m} / \mathrm{s}$, and $v_{\mathrm{G} 3 \mathrm{~B} 2}<v_{\mathrm{G} 4 \mathrm{~B} 1}<v_{\mathrm{GBCC}}$. The experimental results are consistent with the simulation results, showing the same decreasing trend. Compared with the GBCC uniform lattice material, the spreading velocity of stress in the G4B1 and G3B2 heterogeneous lattice materials is reduced by $14.3 \%$ and $33.3 \%$. Compared with the G4B1 heterogeneous lattice material, the spreading velocity of stress of the G3B2 heterogeneous lattice sample is reduced by $14.2 \%$.

Limited by the experimental conditions, the impact quality and speed are inconsistent with the simulation conditions, resulting in a deviation in the experimental results, as shown in Figure 10. However, the changing trend of experimental data and simulation data is consistent with each other. The results show that, compared with the GBCC uniform lattice material, the G4B1 and G3B2 heterogeneous lattice materials can effectively reduce the spreading velocity of stress. It indicates that the number of layers of reinforcement is an important factor affecting the propagation of stress wave in heterogeneous lattice materials. The spreading velocity of stress in heterogeneous lattice materials can be reduced by increasing the number of layers of reinforcement.

5.3. Strain Energy. According to the energy conversation law, the greater the energy absorbed by the lattice materials, the smaller the impact energy absorbed by the transmission rod. The strain energy of the transmitted bar reflects the energy absorbed by the lattice material. The area surrounded by the stress-strain curve is equal to the strain energy. The strain energy can be calculated from the stress-strain integral. The strain energy formula is as follows [42]:

$$
\left\{\begin{array}{l}
Q_{a}=\int_{0}^{\varepsilon} \sigma \mathrm{d} \varepsilon, \\
Q_{T}=\frac{A E^{2}}{\rho C} \int_{0}^{t} \varepsilon_{T}^{2}(t) \mathrm{d} t .
\end{array}\right.
$$

The stress-strain curve of the transmitted bar is shown in Figure 9(c). It can be seen from Figure 9(c) that the stressstrain curves of the transmitted bar of the four lattice samples have the same trend, but the area surrounded by the stressstrain curve is quite different. The strain energy curve is shown in Figure 9(d). During the impact process, the strain energy of the transmitted bar of three lattice samples increases gradually and finally reaches a stable value. The strain energy peaks of the transmitted bar are as follows: $Q_{T-\mathrm{GBCC}}=257.95 \mathrm{~mJ}$, $Q_{T-\mathrm{G} 4 \mathrm{~B} 1}=213.07 \mathrm{~mJ}$, and $Q_{T-\mathrm{G} 3 \mathrm{~B} 2}=181.36 \mathrm{~mJ}$. The relationship between the strain energy peaks is $Q_{T-\mathrm{G} 3 \mathrm{~B} 2}<Q_{T-\mathrm{G} 4 \mathrm{~B} 1}<Q_{T-\mathrm{GBCC}}$. Compared with the GBCC uniform lattice material, the strain energy peaks of the transmitted bars of the G4B1 and G3B2 heterogeneous lattice 


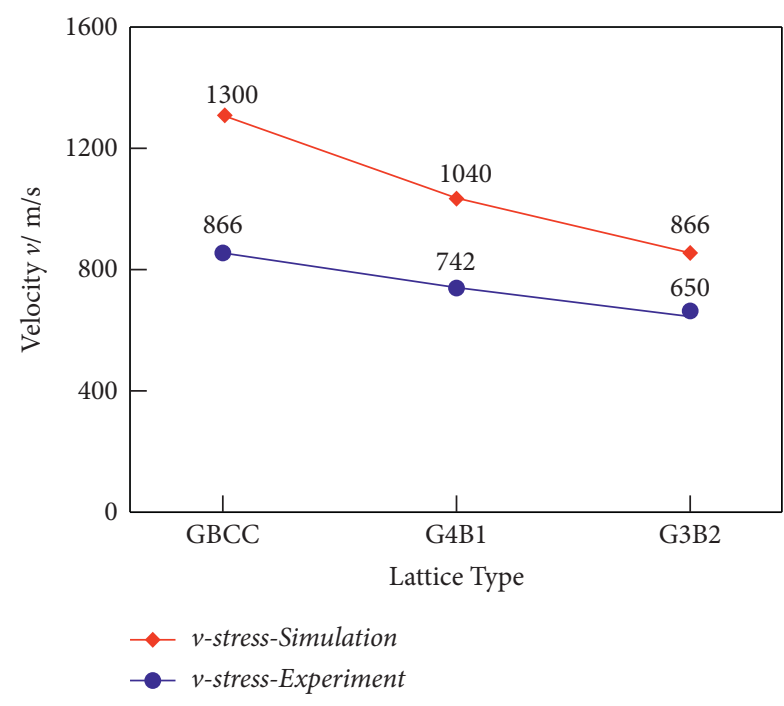

FIgURE 10: The average velocity of stress wave in lattice samples.

materials are reduced by $17.4 \%$ and $29.7 \%$. Compared with the G4B1 heterogeneous lattice material, the stress peak of the transmitted bar of the G3B2 heterogeneous lattice material is reduced by $14.8 \%$.

According to the energy conservation law, the less the strain energy generated by the transmitted bar, the greater the energy absorbed by the lattice materials. The comparison between Figures $7(\mathrm{~b})$ and $9(\mathrm{~d})$ shows that the experimental results are consistent with the simulation results, which proves the optimization trend of energy absorption. The results show that the energy absorption capacity of the G4B1 and G3B2 heterogeneous lattice materials is better than the GBCC uniform lattice material. And the energy absorption capacity of the G3B2 heterogeneous lattice material with two layers of reinforcement is better than the G4B1 heterogeneous lattice materials with a layer of reinforcement. It indicates that the energy absorption properties of heterogeneous lattice materials can be improved by increasing the number of layers of reinforcement.

\section{Conclusions}

In this work, with reference to the composite structure, a heterogeneous lattice material composed of different cells is proposed. The stress wave propagation and energy absorption properties of heterogeneous lattice materials under impact load are analyzed by simulation and SHPB experiment. The experimental results are consistent with the simulation results and prove the optimization trend. The conclusions are as follows:

(1) The stress wave propagation in lattice materials under impact load shows obvious periodic fluctuation and damping vibration trend. Compared with the GBCC uniform lattice material, the spreading velocity of stress in the G4B1 and G3B2 heterogeneous lattice material is reduced by $16.6 \%$ and $18.1 \%$, and the impact time is prolonged by $11.6 \%$ and
$27.9 \%$. It indicates that the heterogeneous lattice materials are able to reduce the spreading velocity of stress.

(2) Compared with the GBCC uniform lattice material, the stress peaks of the transmitted bars of the G4B1 and G3B2 heterogeneous lattice materials are reduced by $24.7 \%$ and $34.8 \%$ and the strain energy peaks are reduced by $17.4 \%$ and $29.7 \%$. It indicates that the heterogeneous lattice materials are able to improve the energy absorption capacity.

(3) By comparing the stress wave propagation and energy absorption properties of three lattice materials, it is found that changing the cell configuration and arrangement to reduce the spreading velocity of stress is helpful to prolong the impact time and enhance the dissipation effect of lattice materials to improve the energy absorption properties of lattice materials.

(4) Compared with the G4B1 heterogeneous lattice material, the spreading velocity of stress of the G3B2 heterogeneous lattice sample is reduced by $14.2 \%$ and the stress energy peak of the transmitted bar is reduced by $14.8 \%$. It indicates that the number of layers of reinforcement is an important factor affecting the stress wave propagation and energy absorption properties of heterogeneous lattice materials.

This work is a reference for the structural innovation and application of heterogeneous lattice materials.

\section{Data Availability}

The data used to support the findings of this study are available from the corresponding author upon request.

\section{Conflicts of Interest}

The authors declare that they have no known conflicts of financial interest or personal relationships that could have appeared to influence the work reported in this paper.

\section{Acknowledgments}

The authors would like to thank the National University of Defense Technology for providing good research and experimental conditions. This research was substantially supported by the National Natural Science Foundation of China (no. 51505488).

\section{References}

[1] X. Cao, X. Ren, T. Zhao, Y. Li, D. Xiao, and D. Fang, "Numerical and theoretical analysis of the dynamic mechanical behaviour of a modified rhombic dodecahedron lattice structure," International Journal of Mechanics and Materials in Design, vol. 17, no. 2, pp. 271-283, 2021.

[2] M. Benedetti, A. D. Plessis, R. O. Ritchie, M. Dallago, S. M. J. Razavi, and F. Berto, "Architected cellular materials: a review on their mechanical properties towards fatigue- 
tolerant design and fabrication," Materials Science and Engineering: R: Reports, vol. 144, Article ID 100606, 2021.

[3] H. Yang, X. Cao, B. Liao, G. Wang, Z. Huang, and Y. Li, "Mechanical properties of bio-molecular inspired MnL2n cage-lattices: simulations \& experiments," Materials Science and Engineering: A, vol. 826, pp. 0921-5093, Article ID 141991, 2021.

[4] V. N. Hoang, P. Tran, V. T. Vu, and H. Nguyen-Xuan, "Design of lattice structures with direct multiscale topology optimization," Composite Structures, vol. 252, Article ID 112718, 2020.

[5] T. Mukhopadhyay, S. Adhikari, and A. Batou, "Frequency domain homogenization for the viscoelastic properties of spatially correlated quasi-periodic lattices," International Journal of Mechanical Sciences, vol. 150, pp. 784-806, 2019.

[6] W. Yu, B. S. Li, Y. Yu et al., "Lattice structure design optimization coupling anisotropy and constraints of additive manufacturing," Materials \& Design, vol. 196, Article ID 109089, 2020

[7] D. Li, R. Qin, B. Chen, and J. Zhou, "Analysis of mechanical properties of lattice structures with stochastic geometric defects in additive manufacturing," Materials Science and Engineering A, vol. 822, pp. 0921-5093, Article ID 141666, 2021.

[8] B. K. Nagesha, V. Dhinakaran, M. V. Shree, K. P. Manoj Kumar, D. Chalawadi, and T. Sathish, "Review on characterization and impacts of the lattice structure in additive manufacturing," Materials Today: Proceedings, vol. 21, no. 01, p. 21, 2019.

[9] B. Wla, B. Hfa, C. Yb, and F. Yc, "Plastic displacement and energy absorption of polycrystalline-like lattice structuresScienceDirect," Materials \& Design, vol. 198, Article ID 109321, 2020.

[10] X. Cui, L. Zhao, Z. Wang, H. Zhao, and D. Fang, "A lattice displacement based model of metallic lattice sandwich plates subjected to impulsive loading," International Journal of Solids and Structures, vol. 49, no. 19-20, pp. 2854-2862, 2012.

[11] R. K. Narisetti, M. Ruzzene, and M. J. Leamy, "Study of wave propagation in strongly nonlinear periodic lattices using a harmonic balance approach," Wave Motion, vol. 49, no. 2, pp. 394-410, 2012.

[12] C. J. Shen, T. X. Yu, and G. Lu, "Double shock mode in graded cellular rod under impact," International Journal of Solids and Structures, vol. 50, no. 1, pp. 217-233, 2013.

[13] G. Zhao, "Modeling stress wave propagation in rocks by distinct lattice spring model," Journal of Rock Mechanics and Geotechnical Engineering, vol. 6, no. 4, pp. 348-355, 2014.

[14] M. R. Karamooz Ravari, M. Kadkhodaei, M. Badrossamay, and R. Rezaei, "Numerical investigation on mechanical properties of cellular lattice structures fabricated by fused deposition modeling," International Journal of Mechanical Sciences, vol. 88, pp. 154-161, 2014.

[15] A. Asadpoure and L. Valdevit, "Topology optimization of lightweight periodic lattices under simultaneous compressive and shear stiffness constraints," International Journal of Solids and Structures, vol. 60-61, pp. 1-16, 2015.

[16] C. Qiu, S. Yue, N. J. E. Adkins et al., "Influence of processing conditions on strut structure and compressive properties of cellular lattice structures fabricated by selective laser melting," Materials Science and Engineering: A, vol. 628, pp. 188-197, 2015.

[17] S. Mukherjee, F. Scarpa, and S. Gopalakrishnan, "Phononic band gap design in honeycomb lattice with combinations of auxetic and conventional core," Smart Materials and Structures, vol. 25, no. 5, Article ID 054011, 2016.
[18] J. Zheng, Q. Qin, and T. J. Wang, "Impact plastic crushing and design of density-graded cellular materials," Mechanics of Materials, vol. 94, pp. 66-78, 2016.

[19] N. Novak, M. Vesenjak, and Z. Ren, "Computational simulation and optimization of functionally graded auxetic structures made from inverted tetrapods," Physica Status Solidi (B), vol. 254, no. 12, Article ID 1600753, 2017.

[20] T. Kim, T. Xie, W. S. Jung, and B. N. Popov, "Development of ultra-low highly active and durable hybrid compressive platinum lattice cathode catalysts for polymer electrolyte membrane fuel cells," International Journal of Hydrogen Energy, vol. 42, no. 17, pp. 12507-12520, 2017.

[21] S. Y. Choy, C.-N. Sun, K. F. Leong, and J. Wei, "Compressive properties of functionally graded lattice structures manufactured by selective laser melting," Materials \& Design, vol. 131, pp. 112-120, 2017.

[22] A. E. H. Hani, A. Bhattacharyya, and D. Maurice, "Modeling of thermal and lattice misfit stresses within a thermal barrier coating," Mechanics of Materials, vol. 122, pp. 159-170, 2018.

[23] A. Bhattacharyya and D. Maurice, "On the evolution of stresses due to lattice misfit at a Ni-superalloy and YSZ interface," Surfaces and Interfaces, vol. 12, pp. 86-94, 2018.

[24] H. Babaei and V. I. Levitas, "Phase-field approach for stressand temperature-induced phase transformations that satisfies lattice instability conditions. Part 2. simulations of phase transformations Si I $\leftrightarrow$ Si II," International Journal of Plasticity, vol. 107, pp. 223-245, 2018.

[25] K. Zhang, C. Zhao, J. Luo, and Y. Ma, "Analysis of temperature-dependent wave propagation for programmable lattices," International Journal of Mechanical Sciences, vol. 171, p. 105372, 2019.

[26] Z. Zhu, Z. Deng, S. Tong, B. Ding, and J. Du, "Elastic wave propagation in hierarchical lattices with convex and concave hexagons stacked vertexes," Journal of the Acoustical Society of America, vol. 146, no. 3, pp. 1519-1527, 2019.

[27] K. Zhang, Y. Su, P. Zhao, and Z. Deng, "Tunable wave propagation in octa-chiral lattices with local resonators," Composite Structures, vol. 220, pp. 114-126, 2019.

[28] M. G. Isaenkova, A. V. Yudin, A. E. Rubanov, A. V. Osintsev, and L. A. Degadnikova, "Displacement behavior modelling of lattice structures manufactured by a selective laser melting of 316L steel powder," Journal of Materials Research and Technology, vol. 9, no. 6, pp. 15177-15184, 2020.

[29] Y. Guo, J. Zhang, L. Chen et al., "Displacement behaviors and energy absorption of auxetic lattice cylindrical structures under axial crushing load," Aerospace Science and Technology, vol. 98, Article ID 105662, 2020.

[30] D. Karličić, B. Mc, A. Tc, and A. Sa, "Wave propagation in mass embedded and pre-stressed hexagonal lattices," Composite Structures, vol. 256, Article ID 113087, 2020.

[31] K. Zhang, P. Zhao, C. Zhao, F. Hong, and Z. Deng, "Study on the mechanism of band gap and directional wave propagation of the auxetic chiral lattices," Composite Structures, vol. 238, Article ID 111952, 2020.

[32] Y. Sun and Q. M. Li, "Dynamic compressive behaviour of cellular materials: a review of phenomenon, mechanism and modelling," International Journal of Impact Engineering, vol. 112, pp. 74-115, 2018.

[33] M. I. Okereke, A. I. Akpoyomare, and M. S. Bingley, "Virtual testing of advanced composites, cellular materials and biomaterials: a review," Composites Part B: Engineering, vol. 60, no. 2, pp. 637-662, 2014.

[34] M. Doroszko, A. Falkowska, and A. Seweryn, "Image-based numerical modeling of the tensile displacement behavior and 
mechanical properties of additive manufactured Ti-6Al-4V diamond lattice structures," Materials Science and Engineering: A, vol. 818, pp. 0921-5093, Article ID 141362, 2021.

[35] P. Płatek, J. Sienkiewicz, J. Janiszewski, and F. Jiang, "Investigations on mechanical properties of lattice structures with different values of relative density made from $316 \mathrm{~L}$ by selective laser melting (SLM)," Materials, vol. 13, p. 2204, 2020.

[36] J. Xin, L. G. Xi, and Z. Meng, "Optimal design of three-dimensional non-uniform nylon lattice structures for selective laser sintering manufacturing," Advances in Mechanical Engineering, vol. 10, pp. 1-19, 2018.

[37] S. Ishikawa, K. Tanaka, D. Yano, and S. Kijimoto, "Design of a disc-shaped viscoelastic damping material attached to a cylindrical pipe as a dynamic absorber or Houde damper," Journal of Sound and Vibration, vol. 475, no. 4, Article ID 115272, 2020.

[38] Y. Dammak, J. H. Thomas, and M. H. B. Ghozlen, "Lamb wave propagation in functionally graded piezoelectric material created by internal temperature gradient," Physics Procedia, vol. 70, pp. 122-125, 2015.

[39] J. G. Yu, F. E. Ratolojanahary, and J. E. Lefebvre, "Guided waves in functionally graded viscoelastic plates," Composite Structures, vol. 93, no. 11, pp. 2671-2677, 2011.

[40] B. Kaur and B. Singh, "The effect of rotation on propagation of Rayleigh wave in an incompressible monoclinic elastic solid," Journal of Mechanics, vol. 36, no. 4, pp. 485-495, 2020.

[41] J. J. Andrew, J. Schneider, J. Ubaid, R. Velmurugan, S. Kumar, and N. K. Gupta, "Energy absorption characteristics of additively manufactured plate-lattices under low- velocity impact loading," International Journal of Impact Engineering, vol. 149, no. 1838, Article ID 103768, 2021.

[42] M. Pajak, J. Janiszewski, and L. Kruszka, "Laboratory investigation on the influence of high compressive strain rates on the hybrid fibre reinforced self-compacting concrete," Construction and Building Materials, vol. 227, pp. 116687.1116687.15, 2019. 